\title{
Analysis and control of micro-stepping characteristics of ultrasonic motor
}

(C) The Author(s) 2020. This article is published with open access at link.springer.com and journal.hep.com.cn

\begin{abstract}
Micro-stepping motion of ultrasonic motors satisfies biomedical applications, such as cell operation and nuclear magnetic resonance, which require a precise compact-structure non-magnetization positioning device. When the pulse number is relatively small, the stopping characteristics have a non-negligible effect on the entire stepwise process. However, few studies have been conducted to show the rule of the open-loop stepwise motion, especially the shutdown stage. In this study, the modal differences of the shutdown stage are found connected with amplitude and velocity at the turn-off instant. Changes of the length in the contact area and driving zone as well as the input currents, vibration states, output torque, and axial pressure are derived by a simulation model to further explore the rules. The speed curves and vibration results in functions of different pulse numbers are compared, and the stepwise motion can be described by a two-stage two-order transfer function. A test workbench based on the Field Programmable Gate Array is built for acquiring the speed, currents, and feedback voltages of the startup-shutdown stage accurately with the help of its excellent synchronization performances. Therefore, stator vibration, rotor velocity, and terminal displacements under different pulse numbers can be compared. Moreover, the two-stage two-order model is identified on the stepwise speed curves, and the fitness over $85 \%$ between the simulation and test verifies the model availability. Finally, with the optimization of the pulse number, the motor achieves $3.3 \mu \mathrm{rad}$ in clockwise and counterclockwise direction.
\end{abstract}

Received August 11, 2019; accepted November 3, 2019

Ning CHEN, Jieji ZHENG, Xianliang JIANG, Shixun FAN,

Dapeng FAN (

College of Intelligence Science and Technology, National University of Defence and Technology, Changsha 410073, China

E-mail: fdp@nudt.edu.cn
Keywords ultrasonic motor, stepping characteristics, pulse number control, synchronous acquisition system, precise positioning

\section{Introduction}

Piezoelectric motors use inverse piezoelectric effect to convert electrical energy into mechanical energy. These motors can operate at non-resonant [1-4] or resonant [5-7] state. At the non-resonant state, the motors usually operate by utilizing the deformations of the piezoelectric elements directly [1], whereas at the resonant state, the motors generally utilize the composition of two vibration modes (same or different) to generate elliptical trajectory movement on the driving tip (stator) [1,2]. The motors that employ ultrasonic frequency to work are called ultrasonic motors. These motors transmit power via frictional force at the contact area between the stator and rotor, which originates from the traveling wave generated along the circumferential direction of the stator [8]. Advantageous features such as high stalling torque $[9,10]$, fast response [11], magnetic resonance compatibility [12,13], and high precision motion without magnetization are the motor's strengths because microscopic stepping motion can be achieved by controlling the actuating voltages. Therefore, the ultrasonic motor is widely used in precise manufacturing, robots, and telescope zoom lens [14-16].

The precise stepping motion can be achieved not only by the position closed-loop control but also in the open-loop mode. The analysis of the open-loop stepwise motion is more meaningful when space is insufficient to place the precise position sensor or the sensor is expensive, such as cell slice actuators, ear surgical devices [17], and magnetic-compatible haptic interfaces $[18,19]$. As the unit of the open-loop stepwise motion, the startupshutdown process under different pulse numbers must be analyzed in detail.

Prior investigations implemented diverse approaches in the stepping or startup-shutdown motion. Kandare and 
Wallaschek [20] examined the transient process of startup stage through an analytical model, and qualitatively analyzed the dynamic characteristics from the perspectives of force, amplitude, and rotational speed. Whether an external load exists, the transient analysis only considers the startup process but lacks the analysis of the shutdown process. Boumous et al. [21] imported the shearing deformation into the calculation of interaction force; the oscillations in the starting section were reduced in amplitude and breadth with the optimization model. Nakagawa et al. [22] measured the transient vibration of the stator by using a laser Doppler velocimeter and detected the revolving speed during the startup-shutdown process. However, the researchers ignored the evaluation of interaction force and stator/rotor contact. Nakamura et al. [23] proposed a strategy to estimate the load characteristics of the linear ultrasonic motor by measuring its rising time and shutdown time. The approximate friction coefficient was determined, which benefits obtaining the load characteristics without a torque meter. However, the entire time length studied was more than $30 \mathrm{~ms}$, which was beyond the range of precise stepping motion. Shen et al. [24] applied the wavelet transforming method in filtering the signal noise in the startup process and reconstructed the velocity signals. Wu et al. [25] studied the stepping motion through experimental research; their method corrects the deviation of the motor stepping angle through the empirical formula identified by the least square method and realizes the equal stepping operation of the circular traveling-wave ultrasonic motor with the load. Although several actuators are not ultrasonic motors, their open-loop stepping characteristics can also provide many useful references. Wang et al. [5] obtained results where vibration displacements along $Y$ and $Z$ directions extend with the increasing pulse numbers in finite element analysis software, as verified by the measured stepping distances. The forward and reverse displacements under different driving frequencies were compared. However, the researchers missed analyzing the interaction force, which can reveal the in-depth mechanism. Xu et al. [1] investigated the displacement differences of a non-resonant-type piezoelectric motor in forward and reverse; they attributed the discrepancies to errors in manufacturing and assembly. Liu et al. [7] conducted a similar work and proved the positive correlation relationship between stepping displacement and pulse number.

The preceding researches mainly focused on the simulation of the startup process, and few studies have been conducted to show the stopping characteristics. On the one hand, previous studies aimed at continuous rotation or stepwise motion when the stepping displacements were more than $500 \mu \mathrm{rad}$. The area of the stopping curve slightly contributes to the entire displacement increment. On the other hand, few practicable synchronous apparatuses detect signals in the high-frequency domain. When the pulse number is small, the shutdown characteristic shows a direct effect on the final stepwise displacements. This study aims at more precise stepwise displacements when the pulse number is relatively small $(N \leqslant 10)$. The stator vibration and rotor rotation under different pulse numbers are compared. A transient speed model based on two-stage two-order functions is proposed and verified to predict the stepping movements.

This paper is divided into five sections. The startupshutdown stage is evaluated through the analytical model in Section 2. A synchronous experimental setup for obtaining the currents and feedback voltages during the stepwise motion is built in Section 3. A series of performances in startup and shutdown stage is investigated, the two-stage two-order transfer functions are verified by the test results, and precise motions in both directions are achieved in Section 4. The conclusions are provided, and prospective research ideas are proposed in Section 5 .

\section{Theoretical analysis of startup and shutdown process}

\subsection{Free vibration of stator}

A simplified vibration model of the free stator is first adopted to distinguish the discrepancies of modal response under different pulse numbers. An $n$-mode resonant vibration is generated on the stator if the driving frequency is equal to the resonant frequency [26]. When $F_{n}$ is the $n$-mode modal force, $M_{n}$ denotes the $n$-mode modal mass, and $\zeta_{n}$ represents the $n$-mode damping coefficient, the modal response in time region is

$$
\begin{aligned}
u_{\mathrm{s}}(t)= & \frac{F_{n}}{2 \zeta_{n} \omega_{n}^{2} M_{n}} \cos \left(\omega_{n} t\right) \\
& +\frac{F_{n}}{2 \zeta_{n} \omega_{n}^{2} M_{n}} \frac{\mathrm{e}^{-\zeta_{n} \omega_{n} t}}{\sqrt{1-\zeta_{n}^{2}}} \sin \left(\omega_{\mathrm{d}} t+\psi_{\mathrm{s}}\right),
\end{aligned}
$$

where the damped natural frequency and phase difference can be calculated as

$$
\omega_{\mathrm{d}}=\sqrt{1-\zeta_{n}^{2}} \omega_{n}, \psi_{\mathrm{s}}=\arctan \frac{\sqrt{1-\zeta_{n}^{2}}}{\zeta_{n}} .
$$

When the pulse number of the driving voltage is $N, t_{N}$ is the instant when the power is cut off. Therefore, the displacement and velocity of the vibration at the transient time can be expressed as

$$
\begin{aligned}
u_{\mathrm{s}}\left(t_{N}\right)= & \frac{F_{n}}{2 \zeta_{n} \omega_{n}^{2} M_{n}} \cos \left(\omega_{n} t_{N}\right) \\
& +\frac{F_{n}}{2 \zeta_{n} \omega_{n}^{2} M_{n}} \frac{\mathrm{e}^{-\zeta_{n} \omega_{n} t}}{\sqrt{1-\zeta_{n}^{2}}} \sin \left(\omega_{\mathrm{d}} t_{N}+\psi_{\mathrm{s}}\right),
\end{aligned}
$$




$$
\begin{aligned}
v_{\mathrm{s}}\left(t_{N}\right) & =\frac{F_{n}}{2 \zeta_{n} \omega_{n} M_{n}} \sin \left(\omega_{n} t_{N}\right)-\frac{F_{n}}{2 \omega_{n} M_{n}} \frac{\mathrm{e}^{-\zeta_{n} \omega_{n} t}}{\sqrt{1-\zeta_{n}^{2}}} \\
& \cdot \sin \left(\omega_{\mathrm{d}} t_{N}+\psi_{\mathrm{s}}\right)+\frac{F_{n} \mathrm{e}^{-\zeta_{n} \omega_{n} t}}{2 \zeta_{n} \omega_{n} M_{n}} \cos \left(\omega_{\mathrm{d}} t_{N}+\psi_{\mathrm{s}}\right) .
\end{aligned}
$$

Over the turning point, the damped free vibration is assumed for the entire stator during the stopping process. Thus, assuming that the damping coefficient and natural frequency are the same as $\zeta_{n}$ and $\omega_{n}$, respectively, the modal displacement can be depicted as Eq. (5). $A_{\mathrm{p}}$ and $\psi_{\mathrm{p}}$ denote the vibration amplitude and phase shift, respectively.

$$
u_{\mathrm{p}}(t)=A_{\mathrm{p}} \mathrm{e}^{-\zeta_{n} \omega_{n} t} \sin \left(\omega_{\mathrm{d}} t+\psi_{\mathrm{p}}\right) .
$$

As derived from the initial status, the value at the end of the starting procedure is equal to that at the beginning of the shutdown process.

$$
\begin{gathered}
u_{\mathrm{p}}(0)=A_{\mathrm{p}} \sin \left(\psi_{\mathrm{p}}\right)=u_{\mathrm{s}}\left(t_{N}\right) \\
v_{\mathrm{p}}(0)=-A_{\mathrm{p}} \zeta_{n} \omega_{n} \sin \left(\psi_{\mathrm{p}}\right)+a \omega_{\mathrm{d}} \cos \left(\psi_{\mathrm{p}}\right)=v_{\mathrm{s}}\left(t_{N}\right) .
\end{gathered}
$$

Therefore, the fundamental parameters $A_{\mathrm{p}}$ and $\psi_{\mathrm{p}}$ can be calculated by

$$
\begin{aligned}
A_{\mathrm{p}} & =\sqrt{u_{\mathrm{s}}\left(t_{N}\right)+\left[\frac{v_{\mathrm{s}}\left(t_{N}\right)+\zeta_{n} \omega_{n} u_{\mathrm{s}}\left(t_{N}\right)}{\omega_{\mathrm{d}}}\right]^{2}} \\
& =\frac{1}{\omega_{\mathrm{d}}} \sqrt{\omega_{n}^{2} u_{\mathrm{s}}^{2}\left(t_{N}\right)+2 \zeta_{n} \omega_{n} u_{\mathrm{s}}\left(t_{N}\right) v_{\mathrm{s}}\left(t_{N}\right)+v_{\mathrm{s}}^{2}\left(t_{N}\right)}
\end{aligned}
$$

$$
\psi_{\mathrm{p}}=\arctan \frac{\sqrt{1-\zeta_{n}^{2}} \omega_{n} u_{\mathrm{s}}\left(t_{N}\right)}{\zeta_{n} \omega_{n} u_{\mathrm{s}}\left(t_{N}\right)+v_{\mathrm{s}}\left(t_{N}\right)} .
$$

When the $\zeta_{n}$ is located at $[0,1]$, the amplitude satisfies

$$
\begin{gathered}
\frac{1}{\omega_{\mathrm{d}}}\left|\zeta_{n} \omega_{n} u_{\mathrm{s}}\left(t_{N}\right)+v_{\mathrm{s}}\left(t_{N}\right)\right|<A_{\mathrm{p}} \\
<\frac{1}{\omega_{\mathrm{d}}}\left|\omega_{n} u_{\mathrm{s}}\left(t_{N}\right)+v_{\mathrm{s}}\left(t_{N}\right)\right| .
\end{gathered}
$$

Equations (8) and (9) show that the amplitude and phase shift of the stopping function are all connected with $\zeta_{n} \omega_{n} u_{\mathrm{s}}\left(t_{N}\right)+v_{\mathrm{s}}\left(t_{N}\right)$, which implies that the shutdown speed curves are related to the modal response and speed at the shutdown moment. Moreover, when the turn-off point is in the stabilized stage, the second item of Eq. (3) is maintained at zero. We substitute Eqs. (3) and (4) into Eqs. (8) and (9), and the amplitude and phase difference can be transformed into

$$
\begin{gathered}
A_{\mathrm{p}}=\frac{F_{n}}{2 \zeta_{n} \omega_{n}^{2} M_{n}} \sqrt{1+2 \zeta_{n} \cos \left(\omega_{n} t_{N}\right) \sin \left(\omega_{n} t_{N}\right)} \frac{1}{\sqrt{1-\zeta_{n}^{2}}}, \\
\psi_{\mathrm{p}}=\arctan \frac{\sqrt{1-\zeta_{n}^{2}} \cos \left(\omega_{n} t_{N}\right)}{\sin \left(\omega_{n} t_{N}\right)+\zeta_{n} \cos \left(\omega_{n} t_{N}\right)} .
\end{gathered}
$$

The decay rule of modal amplitude is directly related to the function $\sqrt{1+2 \zeta_{n} \cos \left(\omega_{n} t_{N}\right) \sin \left(\omega_{n} t_{N}\right)}$, and the conditions can be discussed as follows [12]:

where $k=0,1,2, \ldots$

The preceding assumptions are based on the performances in the driving frequency close to the resonance frequency. However, the region where the ultrasonic motor usually works exceeds the resonance frequency, and the vibration of the stator shows specific changes after the friction layer. Thus, the function derived above is not entirely equal to the speed of the rotor, and a more accurate analytical model is still needed when parsing the rotor speed.

\subsection{Division of startup and shutdown process}

Figure 1 illustrates the working principle of the travelingwave ultrasonic motor (TRUM). Piezoelectric ceramics are actuated by the two-phase sinusoidal voltages $\left(U_{\mathrm{A}}\right.$ and $\left.U_{\mathrm{B}}\right)$ determined by the amplitude $\left(U_{\mathrm{m}}\right)$, frequency $(f)$, and phase difference $(\alpha)$, as shown in Fig. 1(a). The twochannel input currents $I_{\mathrm{A}}$ and $I_{\mathrm{B}}$ can be informed by linking to the electrical network of the piezoelectric ceramics. The stator vibrates with the amplitude $A_{\text {sat }}$ during the energy, as shown in Fig. 1(b). The modal responses of Phases $\mathrm{A}$ and $\mathrm{B}$ are characterized as $q_{\mathrm{A}}$ and $q_{\mathrm{B}}$, respectively. The circumferential rotation of the stator, which is propelled by the traveling wave, drives the rotor through the friction force. In Fig. 1(c), $F_{\text {pre }}$ represents the preload force, and $F_{z}$ means the vertical force acting on the rotor. The output torque $T_{\text {out }}$ is produced to overcome the applied torque $T_{\text {load}}$, as shown in Fig. 1(d). The entire rotor's mass and inertia are $M_{\mathrm{r}}$ and $J_{\mathrm{r}}$, respectively. transformation from electrical energy to mechanical 
(a)

(b)

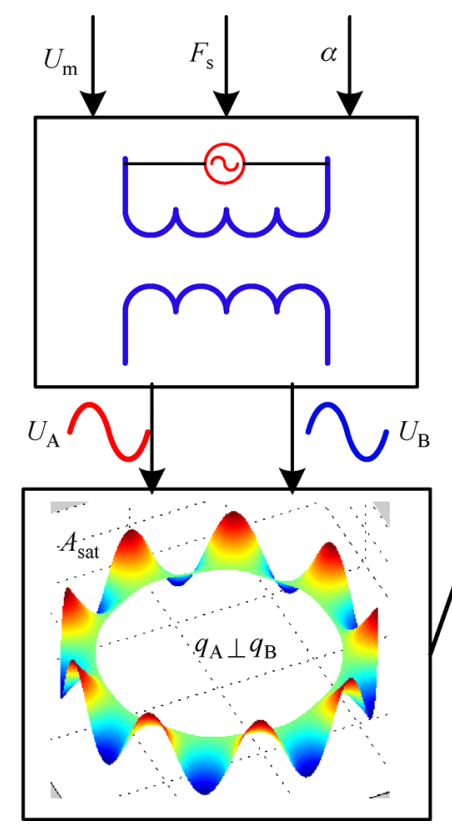

(c)

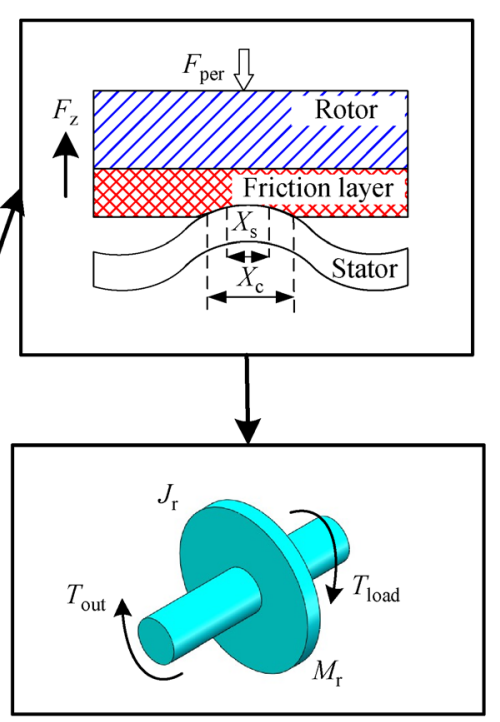

Fig. 1 Block diagram of TRUM: (a) Driver; (b) PZT/stator vibration; (c) stator/rotor contact; and (d) rotor with external load.

The contact diagram is shown in Fig. 1(c). Only the finite area of every traveling wave is embedded into the friction material, and the contact area can be separated into driving and braking zones. The separating points where the rotor velocity is equal to the stator surface velocity are called no-slip points. In this paper, the contact length is defined as $X_{\mathrm{c}}$, and the driving length is determined as $X_{\mathrm{d}}$. The two parameters yield $0 \leqslant X_{\mathrm{d}} \leqslant X_{\mathrm{c}} \leqslant 40$, and their values can be expressed as

$$
\begin{gathered}
X_{\mathrm{c}}=\frac{20}{\pi} \arccos \frac{h-z(t)}{R_{\mathrm{av}} A_{\mathrm{sta}}}, \\
X_{\mathrm{d}}=\frac{20}{\pi} \arccos \frac{\Omega_{\mathrm{r}} R_{0}^{2}}{\lambda h R_{\mathrm{av}} A_{\mathrm{sta}} f},
\end{gathered}
$$

where $z(t)$ reflects the vertical displacement of the rotor, $\lambda$ represents the wavelength of the traveling wave, $R_{0}$ represents the average radius of the stator, $h$ denotes the half-length of the stator-ceramic laminated plate, and $\Omega_{\mathrm{r}}$ is the angular velocity of the rotor. When $R(r)$ is defined as the transverse displacement distribution function along the radial direction, the value at the average radius is defined as $R_{\mathrm{av}}$.

Similar to the model proposed by Ref. [27], our model combines the equivalent circuit and ontology motor model. When 800 pulses are injected into the model, the main electric-mechanical parameters in a specific stepwise process can be obtained, as shown in Fig. 2. The motor's pre-pressure is $300 \mathrm{~N}$. Driving amplitude, frequency, and phase difference are $200 \mathrm{~V}, 43 \mathrm{kHz}$, and $90^{\circ}$, respectively. The time axis in Fig. 2 shows that the entire startupshutdown procedure can be divided into the following five stages according to different vibration statuses:
(1) Pre-static stage: $\left[T_{0}, T_{1}\right]$

Once the power is switched on, the actuating voltage amplitude jumps from zero to a specific value immediately while the currents fluctuate, as shown in Fig. 2(a). However, the vibration amplitude of the stator is so small that it is unable to generate a valid traveling wave to propel the stator (Fig. 2(b)). The interaction force cannot overcome the static friction and damping force, which makes $X_{\mathrm{c}}$ equal to $40^{\circ}$. Thus, the contact zone stretches over the entire wavelength of the traveling wave, which results in the motionless rotor.

(2) Dynamic friction and fluctuation stage: $\left[T_{1}, T_{2}\right]$

As the process continues, the energy accumulated in the pre-static stage provides impetus to the persistence circumferential vibration until the driving force conquers the static friction. In succession, the contact zone becomes narrower, and the contact points and stick points depart from each other gradually, as observed in Fig. 2(f). When the motor starts, the contact angle is maintained at $40^{\circ}$ until the $M$ point arrives, which lags the change of the driving zone. Equation (15) shows that the length of the driving zone is directly connected to the revolving speed. However, the conclusion is only proven in the time domain $\left[T_{\mathrm{p}}, T_{2}\right]$ between Figs. 2(c) and 2(f). The contact points need time to step over the neutral layer of the piezoelectric laminated plate. The output torque and dynamic vertical force experience similar fluctuations, which are not only the results of the vibration but also the source of the motion for the next time slice, as shown in Figs. 2(e) and 2(f).

(3) Stabilized stage: $\left[T_{2}, T_{3}\right]$

As the name implies, the amplitude of the vibration stabilizes at this stage; so do the rotor speed and driving 
force. Therefore, the balance between the driving zones and braking zones is achieved. The contact length is $29.5^{\circ}$, and the driving length is $10.1^{\circ}$, as shown in Fig. 2(f). Under actual operation conditions, the velocity decreases due to the increasing heat dissipation from temperaturesensitive piezoelectric materials.

(4) Vibration decay stage: $\left[T_{3}, T_{4}\right]$

This stage occurs when the driving voltages are withdrawn. The currents mutate immediately while the modal displacement attenuates slowly due to the damping factor in the motor. Figure 2(c) shows that the rotor velocity falls in accordance with the vibration continuously. The contact length extends and returns to the inertial value gradually.

(5) Self-locking stage: $\left[T_{4}, T_{5}\right]$

Even though the voltage drops to a minute scale, the stator vibrates on a microscopic scale due to the inertial effect and damping. The motor should vibrate around the zero speed after crossing the zero line. However, the simulation fails to present this phenomenon, which may be due to the simplified interaction assumption.

Based on the above results, the ultrasonic motor goes through five stages from the power-on moment to the selflocking time due to the different vibration statuses. Before the speed stabilizes, the contact length and driving length change in the intricate laws, which may result in different stepwise characteristics when the pulse number varies.

\subsection{Factors affecting stepping resolution}

Several factors affect the terminate stepper resolution stretch over the entire five stages of the startup-shutdown process. The controllable variables of the motor to achieve the stepwise movement in open-loop mode include amplitude, frequency, phase difference, and pulse number.

Whether the amplitude or frequency affects the vibration and contact states, they affect the stepwise resolution with the complicated mapping relationship. Therefore, uncertainty and complexity make these two parameters unsuitable for adjusting the stepwise resolution effectively and accurately. Keeping the amplitude and frequency constant becomes the primary work before the control of stepwise displacement. Moreover, the actuating frequency can be optimized through the method proposed by Refs. $[28,29]$, which confirms the optimum working frequency based on the minimum power consumption. Thus, the supplying power of the motor driver can be limited to a small value, which benefits the intergradation of the motor and driver. For the phase difference, the moving direction is investigated when the phase shift is changed from $-90^{\circ}$ to $90^{\circ}$. However, even when other parameters are the same, several discrepancies remain in the stepping resolution between clockwise $(\mathrm{CW})$ and counterclockwise $(\mathrm{CCW})$ directions due to the errors in manufacturing and assembling, which will be investigated in the experimental process. If the two-phase voltages are in a non-orthogonal state, the distorted traveling wave will cause the elusive elliptic trajectories of stator particles. What's worse, the modal mixture is doped in the vibration of the stator, and the intense complexity makes the stepwise motion unpredictable.

When we exclude the plan to change the driver parameters, controlling the pulse number is the only effective way to obtain different displacements. Given that the pulse number changes at the same amplitude and frequency, pre-static stage, dynamic friction \& fluctuation
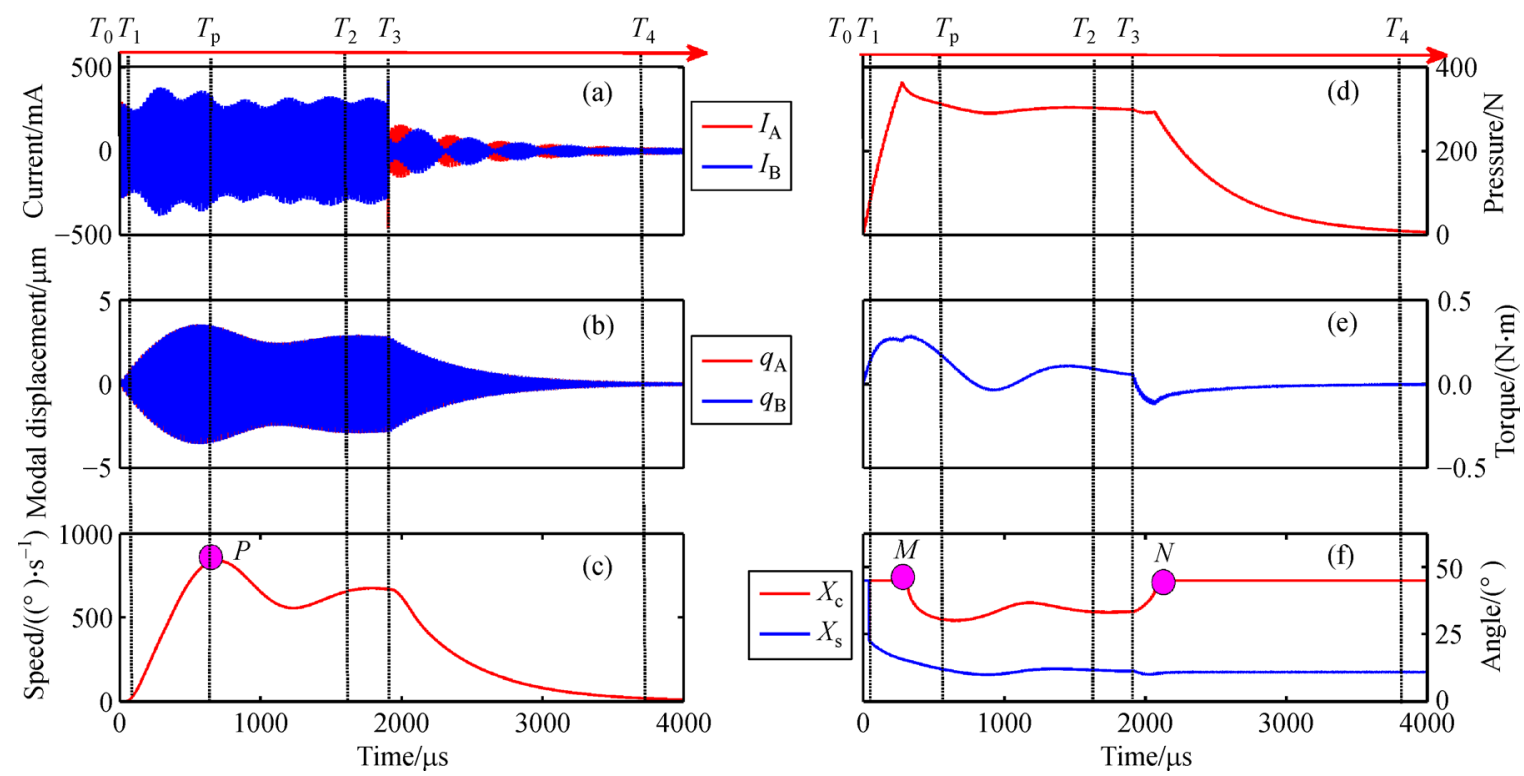

Fig. 2 Transient response of unloaded motor after 800 pulse activations: (a) Generated currents $I_{\mathrm{A}}$ and $I_{\mathrm{B}}$, (b) modal amplitude of two standing waves, (c) revolving speed of rotor, (d) vertical pressure $F_{z}$, (e) output torque $T_{\text {out }}$, and (f) width of contact and driving zone. 
stage, and stabilized stage of the curves are superimposed on each other. Finally, the curve variables in the vibration decaying stage, especially the damping parameters, must be predicted.

\subsection{Simplified speed model with different pulse numbers}

The speed curves under different excitation numbers are described in Fig. 3. The velocity curves in terms of varying pulse counts show different turn-off characteristics, and they eventually converge to the same turn-off self-locking state with different attenuation slopes. After reaching the cut-off point, each velocity curve exhibits a slight increase to the peak value before the continuous decline because of the inertial effect of the rotor in the initial process. Figure 4 shows that more refined observations of the modal displacements guide us in finding the amplitude differences with respect to different pulse numbers. When the number $N \leqslant 30$, the stator amplitude $A_{\text {sta }} \leqslant 3.8 \mu \mathrm{m}$. As the number of sinusoidal signals increases, the modal curves are superimposed slightly along the time axis until the supply power is turned off. After crossing the cutoff point, the modal responses undergo similar attenuating patterns.

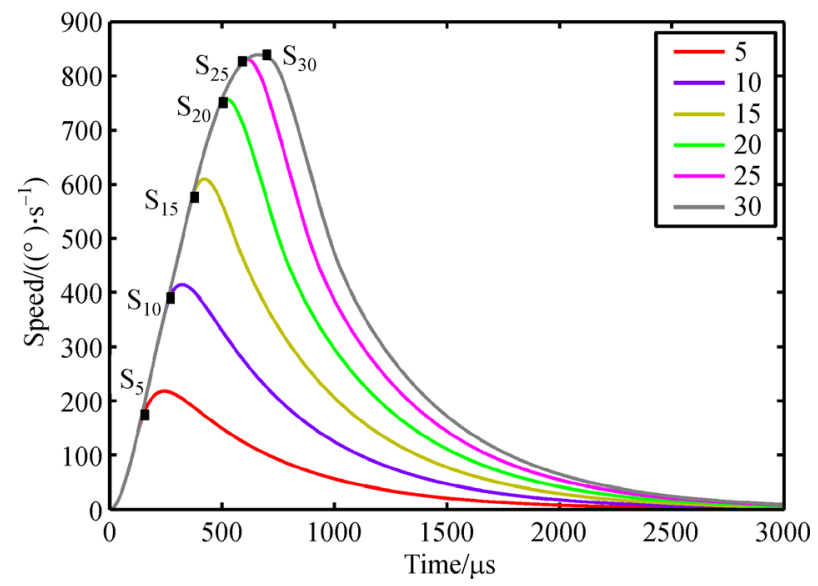

Fig. 3 Simulation speed under different pulse numbers.

Figure 4 shows that the location of the on-off switch points can be marked by $S_{N}$, where $N$ denotes the pulse number. According to different dynamic characteristics of the startup and shutdown stages, the description function of startup and shutdown can be expressed as Eqs. (16) and (17), respectively. The coefficients of the starting section and shut-off section for the rotor are $\left(K_{\mathrm{s}}, \xi_{\mathrm{s}}, \omega_{n \mathrm{~s}}\right)$ and $\left(K_{\mathrm{p}}\right.$, $\left.\xi_{\mathrm{p}}, \omega_{n \mathrm{p}}\right)$, respectively.

$$
\begin{aligned}
G_{\mathrm{s}}(s) & =\frac{K_{\mathrm{s}}}{s^{2}+2 \xi_{\mathrm{s}} \omega_{n \mathrm{~s}} s+\omega_{n \mathrm{~s}}^{2}}, \\
G_{\mathrm{p}}(s) & =\frac{K_{\mathrm{p}}}{s^{2}+2 \xi_{\mathrm{p}} \omega_{n \mathrm{p}}+\omega_{n \mathrm{p}}^{2}} .
\end{aligned}
$$

The damping status from different moments reflect the different dynamic properties of the shutdown process, which may engender the diverse vibration attenuation time. Finally, we can obtain the stepwise angular displacements by calculating the area of the speed curve. $\Omega_{\mathrm{s}}(t)$ and $\Omega_{\mathrm{p}}(t)$ are the respective speeds in the time domain, and the stepwise displacement yields Eq. (18), where $t_{\mathrm{s}}$ and $t_{\mathrm{p}}$ denote the end moment of the startup stage and shutdown stage. $t_{\mathrm{s}}$ is equal to $t_{N}$ in Eq. (3)

$$
S\left(t_{\mathrm{s}}, t_{\mathrm{p}}\right)=\int_{0}^{t_{\mathrm{s}}} \Omega_{\mathrm{s}}(t) \mathrm{d} t+\int_{t_{\mathrm{s}}}^{t_{\mathrm{p}}} \Omega_{\mathrm{p}}(t) \mathrm{d} t .
$$

\section{Experimental setup}

The ultrasonic motor employed for the experimental test is PMR60 (NUAA Super Control Technology Co., Ltd., China). The auxiliary electrode is mounted in addition to the A and B sections, as shown in Fig. 5(a). The electrode arrangement in the ceramic ring is shown in Fig. 5(b). The feedback voltage is obtained from this auxiliary electrode once the mechanical vibrations are generated on the stator [30]. This feedback voltage $U_{\mathrm{F}}$ is proportional to the vibration amplitude of the stator due to the piezoelectric conversion, as shown in Eq. (19), where $K_{\mathrm{fb}}$ represents the conversion factor.

$$
U_{\mathrm{F}}=\frac{\sqrt{2} K_{\mathrm{fb}} A_{\mathrm{sta}} R_{\mathrm{av}} \lambda}{2 \pi} \sin \left(w t+\frac{\pi}{4}\right) .
$$

Figure 6(a) shows an incremental encoder TS5700N8501 (Tamagawa Seiki Co., Ltd., Japan) with resolution of 23 bits per revolution. The encoder resolution reaches $0.75 \mu \mathrm{rad}$. Moreover, owing to its serial communication mode with a $2.5 \mathrm{MHz}$ baud rate, the sampling interval of the encoder can reach $37 \mu \mathrm{s}$; thus, the microscopic details of transient speed can be observed clearly. A Field Programmable Gate Array (FPGA) board (PXIE 7854R, National Instrument Co., Ltd., United States) is adopted because it can generate sinusoidal signals with output frequency of up to $1 \mathrm{MHz}$ and collect currents and voltages with independent sampling rates of up to $750 \mathrm{kHz}$ to accommodate speed and accuracy [31]. The generated sinusoidal signals $\left(U_{\mathrm{A} 0}\right.$ and $\left.U_{\mathrm{B} 0}\right)$ are used for the source of amplifiers and transformers inside the driver. Two-phase driving voltages $\left(U_{\mathrm{A}}\right.$ and $\left.U_{\mathrm{B}}\right)$ are formed to actuate the ultrasonic motor. Figures 6(b) and 6(c) show that the hall sensors (Zhonghuo Sensing Technology Co., Ltd., China) for detecting the input currents are used to acquire the realtime driving currents, and the voltage transformers from the same company are employed to measure the three voltages ( $U_{\mathrm{aa}}, U_{\mathrm{bb}}$, and $\left.U_{\mathrm{ff}}\right)$. Finally, amplitude, frequency, phase difference, and excitation number can be set in the Labview interface integrated with the FPGA board. FPGA's excellent time sequence organizing function 

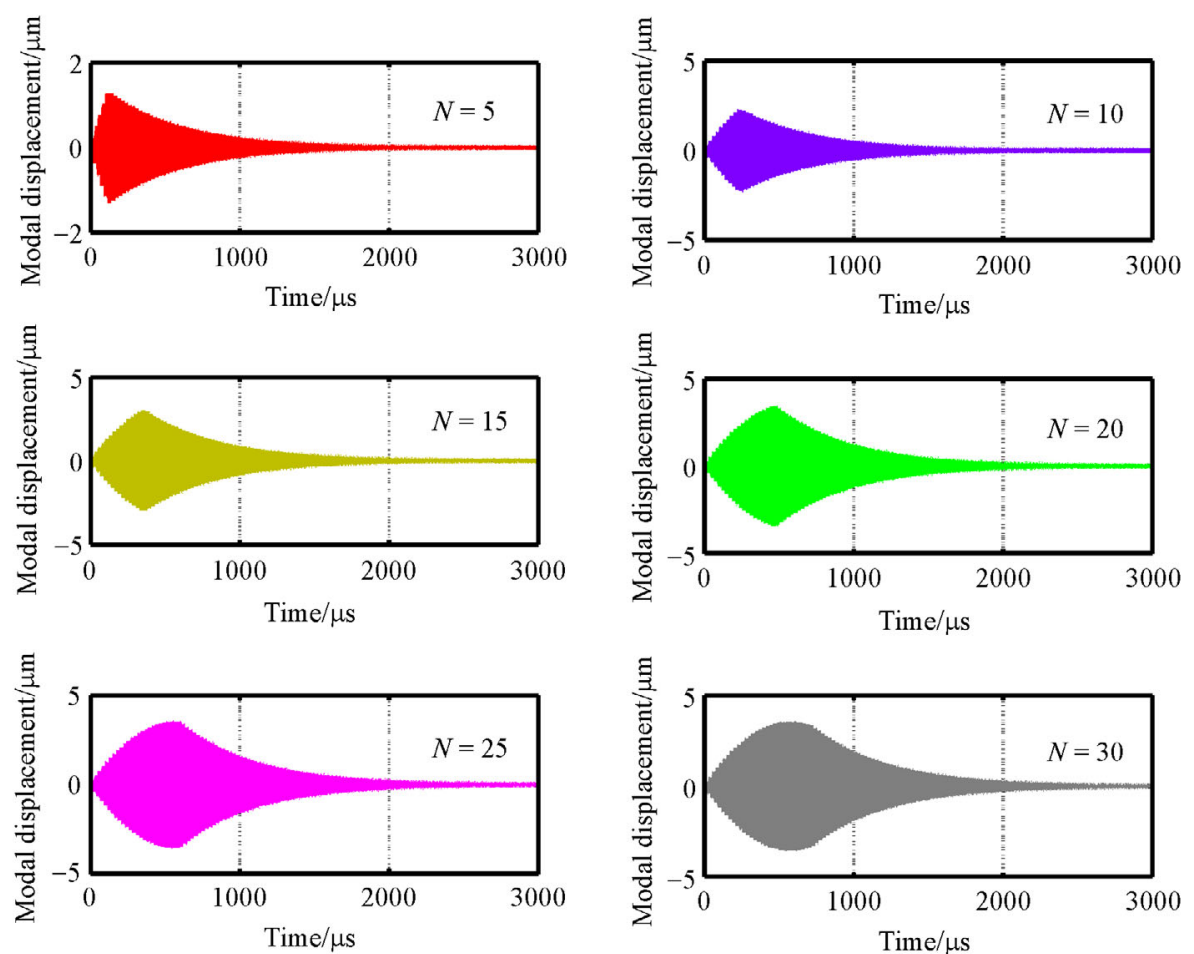

Fig. 4 Vibration mode under different pulse numbers.

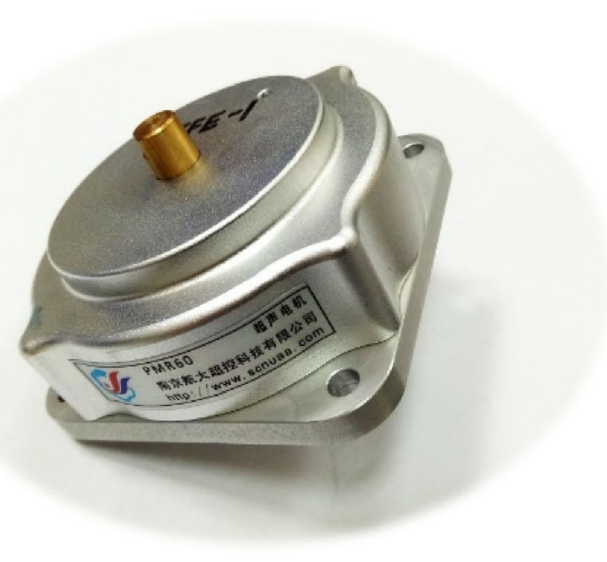

(a)

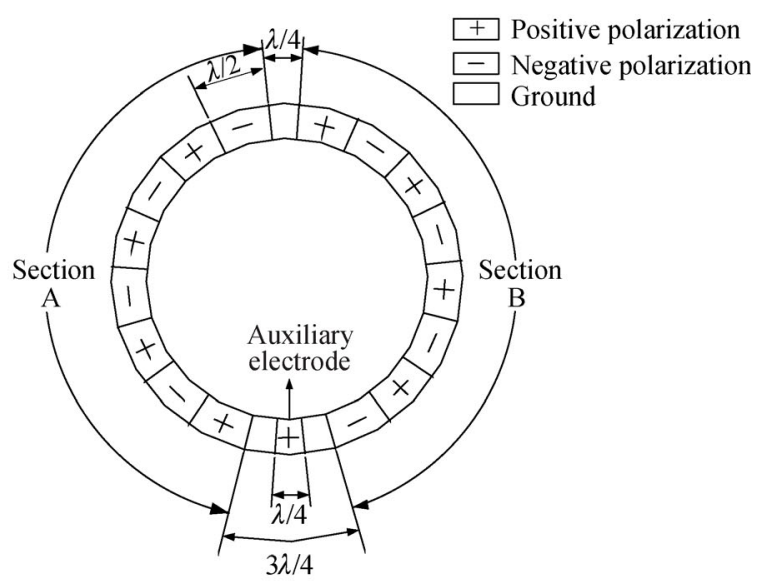

(b)

Fig. 5 TRUM used for experimental test. (a) Picture of PMR60 and (b) electrode arrangement of piezoelectric ring in PMR60.

guarantees the superior synchronization performance of the entire test platform, which ensures reliable analysis of internal laws of the stepwise motion.

\section{Experimental test}

A series of startup-shutdown responses by controlling the driving parameters and pulse numbers is investigated on the built synchronous acquisition system.
4.1 Stepwise angular displacement under different driving parameters

Different mapping relationships are observed between the steady speed and driving parameters, which induce the discrepancies of the stepwise angular displacements. Figure 7 displays the stepping displacements in functions of different driving parameters. The range of amplitude is $[160,240]$, the frequencies are changed from 39 to 43.5 $\mathrm{kHz}$, and all curves are obtained when the pulse number is 


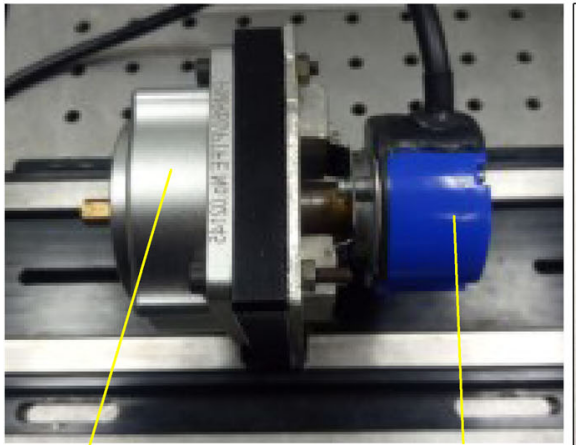

PUM60
Encoder

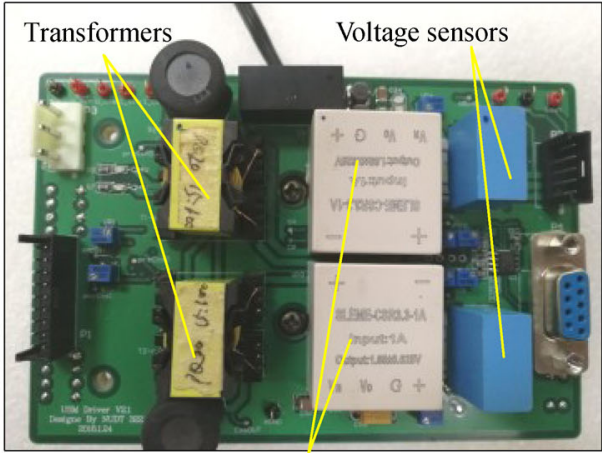

Current sensors

(b)

(a)

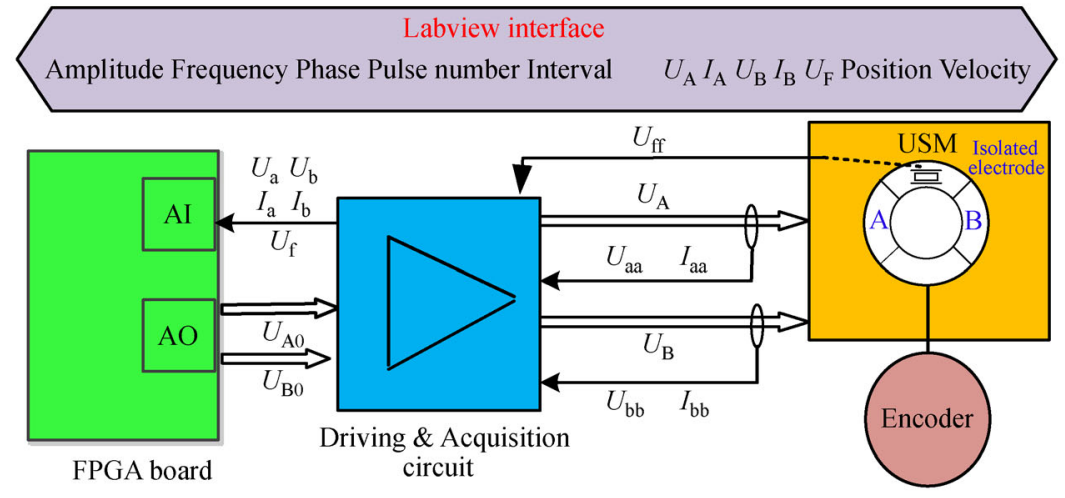

(c)

Fig. 6 Experimental setup: (a) TRUM platform with an incremental encoder, (b) driver with measurement of voltages and currents, and (c) block diagram of experimental setup.

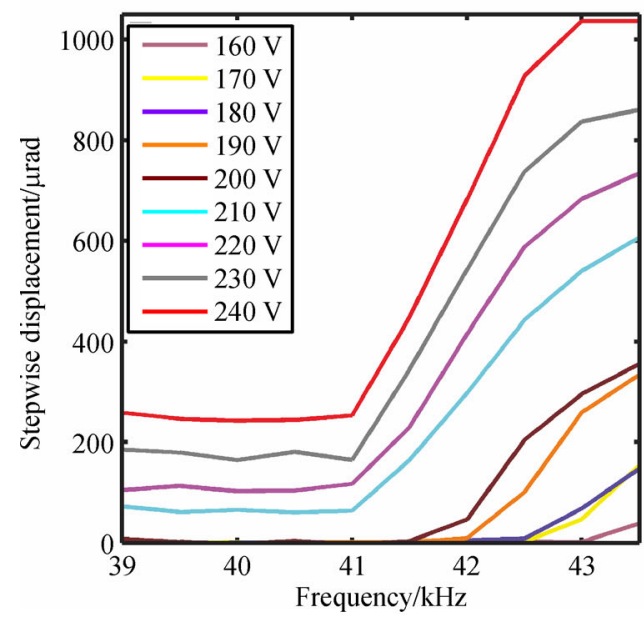

(a)

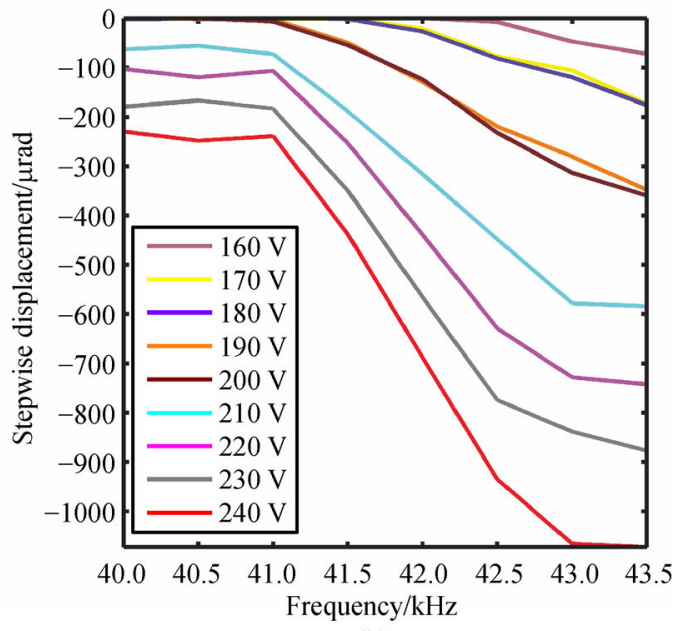

(b)

Fig. 7 Stepwise displacements in functions of different driving variables: (a) Test in CW direction; (b) test in CCW direction.

10. Figure 7(a) reveals the displacements in the $\mathrm{CW}$ direction, whereas Fig. 7(b) demonstrates those in the $\mathrm{CCW}$ direction. The results in forward and reverse are symmetric, whereas the displacements in the $\mathrm{CW}$ direction are more substantial than those in the $\mathrm{CCW}$ direction. The value arises in function of increasing amplitude. Slight differences are observed between angular displacements when the frequency is less than $41 \mathrm{kHz}$. When the frequency increases from 41 to $43.5 \mathrm{kHz}$, the angular displacement generally ascends. As the amplitude is from 
160 to $240 \mathrm{~V}$, the typical test amplitude is $200 \mathrm{~V}$, which is equal to the average of the upper and lower limits. The test frequency for the experiments is $43 \mathrm{kHz}$, which is calculated by the criterion proposed by Ref. [28] to minimize the power consumption and maximize the mechanical quality factor.

\subsection{Reversing features of stepping motion}

According to the motor working principles, the reversal of ultrasonic motor originates from the change of phase difference within the two driving signals in time and space. Figure 8(a) illustrates the circumference position where the excitation number is 5 or 10 for different driving parameters in the $\mathrm{CW}$ and $\mathrm{CCW}$ directions. The amplitude of actuating signals is $200 \mathrm{~V}$ and the driving frequency is $43 \mathrm{kHz}$. Approximately 20\% overshoots occur in every rising stage. Figure 8(b) shows the stepping angular displacements after statistics. The results show that whether rotating in the $\mathrm{CW}$ or $\mathrm{CCW}$ direction, the first position increments at the first time are higher than those in the three succeeding times because the two contract borders are non-asymmetric with respect to the peak of the traveling wave shown in Fig. 1(c), and the contract length is larger on the opposite side than in the semi-depart. When the direction of the traveling wave changes, a backlash-like deformation occurs in the gear reducer. After three-time adjustments, the angular displacements stabilize.

The voltages and currents increase dramatically at the turn-on instant, similar to the vibration of the stator. The test results may differ from the simulation results in Fig. 9. The amplitudes of voltages and currents are the same in the $\mathrm{CW}$ and $\mathrm{CCW}$ directions. However, the magnitudes of the feedback voltage are higher in the $\mathrm{CCW}$ direction, and the decay time varies. The decay time in the $\mathrm{CCW}$ direction is longer, which indicates the vibration difference in the commutating process and may be attributed to the stronger anti-friction effect [32-34] caused by larger vibration amplitude in the CCW direction. With the reduction of friction coefficient, the braking force decreases, and the weakening of the braking effect lengthens the time of motor vibration damping.

\subsection{Speed curves under different pulse numbers}

Few differences are observed in the images of the voltages and currents, so concentrates are more assigned for feedback voltage. The speed results including the starting and stopping processes under different pulse numbers are shown in Fig. 10. Figure 11 illustrates the feedback voltages when the amplitude is $200 \mathrm{~V}$ and the frequency is $43 \mathrm{kHz}$. The figure shows that the waveform is gradually superimposed. Waves with fewer pulse counts are spun off from those with more pulse numbers. Detailed results with pulse numbers $(4,6,8$, and 10) are depicted in Fig. 11(b), where vertical lines with different colors are employed to represent each shutdown moment and explore the detailed vibration characteristics. These curves are the separating lines between the startup and shutdown segments. Furthermore, the phenomenon such as modal mixture appears in every shutdown curve, which makes the vibration curves highly complicated such that they cannot be accurately calculated. The repetition on the speed curve of every startup process is good such that we can obtain the same startup function if the amplitude and frequency remain constant.

\subsection{Identification of stepwise angular displacement}

The least squares identification is implemented for the startup and shutdown stages from the power-off moment to

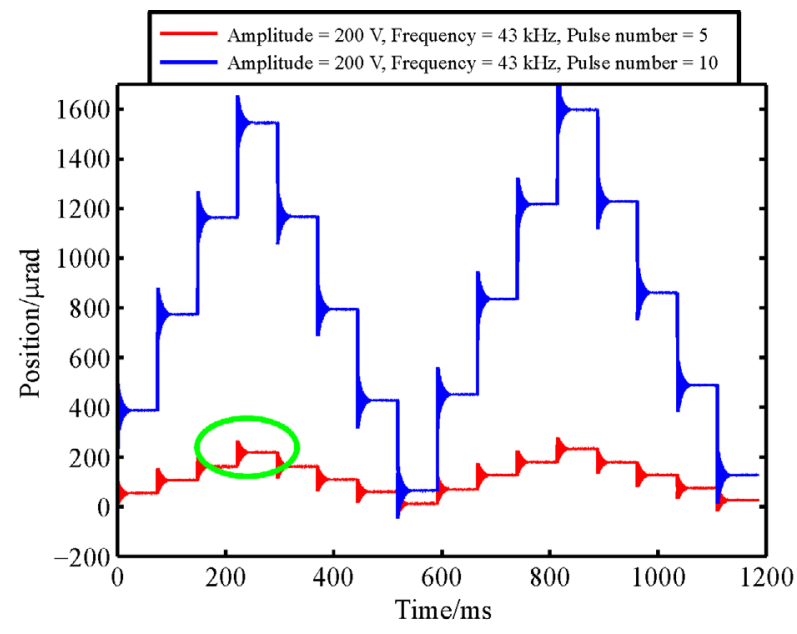

(a)

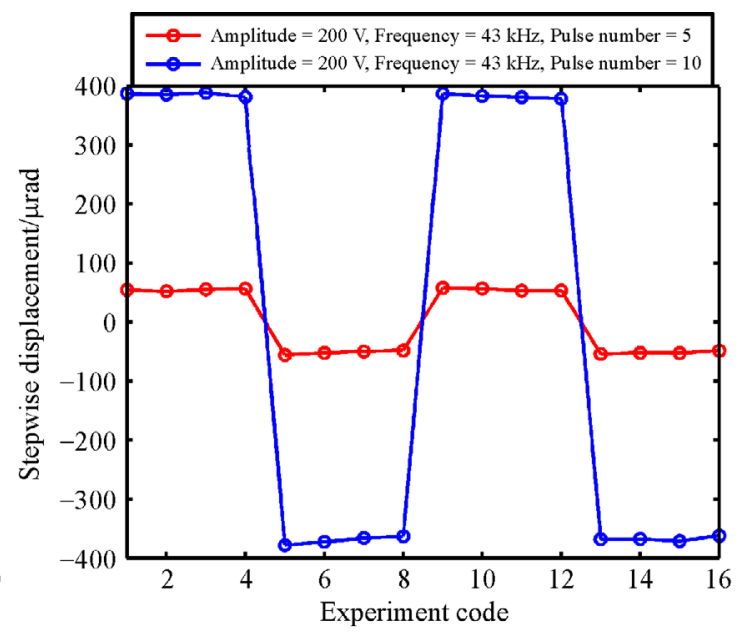

(b)

Fig. 8 Stepwise angular displacement of motion: (a) Stepwise angular position and (b) stepwise displacement. 

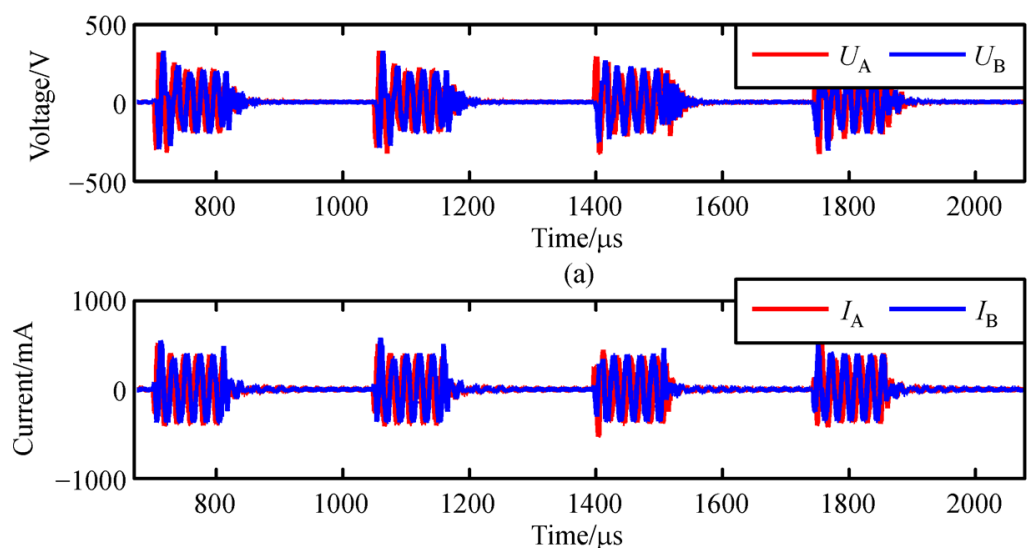

(b)

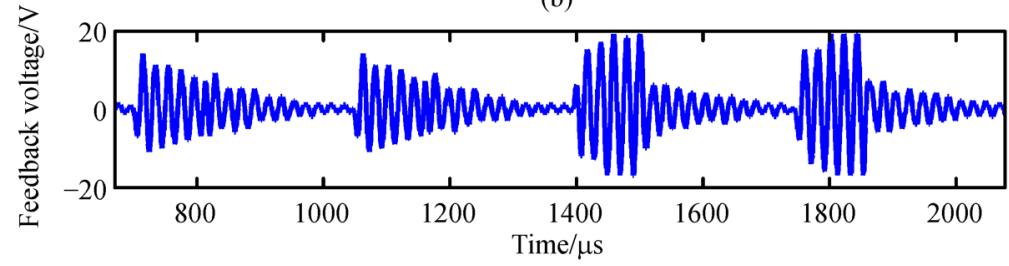

(c)

Fig. 9 Reversing response of PUM60: (a) Input voltages $U_{\mathrm{A}}$ and $U_{\mathrm{B}}$, (b) input currents $I_{\mathrm{A}}$ and $I_{\mathrm{B}}$, and (c) feedback voltage $U_{\mathrm{F}}$.

obtain the transfer functions shown in Eqs. (16) and (17). With the identification of the results when the pulse number is 400, the natural frequencies and damping coefficients identified from the startup process are drawn in Table 1.

The identified parameters of the shutdown process and speed at the shutdown moment are demonstrated in Fig. 12. The comparison results indicate that the parameters are relatively stable within the range of $[1,10]$ and $[100,500]$ because the system within 10 pulses is on the initial rising stage where the velocity does not exceed the steady-state velocity, and the speed value stabilizes after the fluctuations when the excitation number is more extensive than 100. Here the speed at the stopping moment is defined as $v e l_{\text {stop. }}$. Observations in Figs. 12(c) and 12(d) can help us obtain the following statistical conclusions if the pulse number is no more than 10: (1) The identified gain and velocity at the shutdown moment are proportional to the excitation number; (2) the first-order frequency of the stopping stage $\omega_{n \mathrm{p}}$ is approximately $2360 \mathrm{rad} / \mathrm{s}$, which is larger than the similar coefficient in the startup process; and (3) the damping coefficient is approximately 0.013 in both directions.

Furthermore, comparison results of the pair parameters $5 K_{\mathrm{p}} / v e l_{\text {stop }}$ and $\omega_{n \mathrm{p}}^{2}$ reveal that $K_{\mathrm{p}} / v e l_{\text {stop }}$ is strongly proportional to $\omega_{n \mathrm{p}}^{2}$, and the proportional coefficient is approximately 0.197 , as shown in Fig. 13. In conclusion, the identified results can guide us to achieve a clear function at the shutdown stage, which can be briefly described as

$$
\begin{aligned}
G_{\mathrm{p}} & =v e l_{\text {stop }} \frac{K_{\mathrm{p}} / v e l_{\text {stop }}}{s^{2}+2 \xi_{\mathrm{p}} \omega_{n \mathrm{p}} s+\omega_{n \mathrm{p}}^{2}} \\
& =\frac{0.197 \omega_{n \mathrm{p}}^{2} v e l_{\text {stop }}}{s^{2}+2 \xi_{\mathrm{p}} \omega_{n \mathrm{p}} s+\omega_{n \mathrm{p}}^{2}} .
\end{aligned}
$$

Based on the preceding test, natural frequency $\omega_{n \mathrm{~s}}$ and damping coefficient $\xi_{\mathrm{s}}$ are $2360 \mathrm{rad} / \mathrm{s}$ and 0.013 , respectively. More discrete pulse numbers are selected from 2.5 to 9.5 with 0.5 step to prove the validation. The comparison results between the fitting results and experimental ones are shown in Fig. 14. Satisfactory agreements are obtained because the finesses exceed $85 \%$, which suggests the validation of identification and prediction.

We extend the above curves slightly, and the pulse number that can generate the precision stepping movement is searched to satisfy the requirements of stable operation and reliable positioning. Figures 15(a) and 15(b) describe the stepping curves in both directions with the same resolution $(3.3 \mu \mathrm{ad})$. When the motor operates in the openloop pattern, the pulse number is 2.1 in the $\mathrm{CW}$ direction and 2.2 in the CCW direction, respectively. Several differences are observed in each startup stage, but their steady positioning performances are close. If the preliminary pulse numbers are further reduced, starting the motor is a challenging task, and the resolution is not always uniform. This phenomenon is due to insufficient energy accumulation in the startup section. In these cases, the stable and reliable stepping motion image cannot be accomplished. Therefore, it is unsuitable for the final 


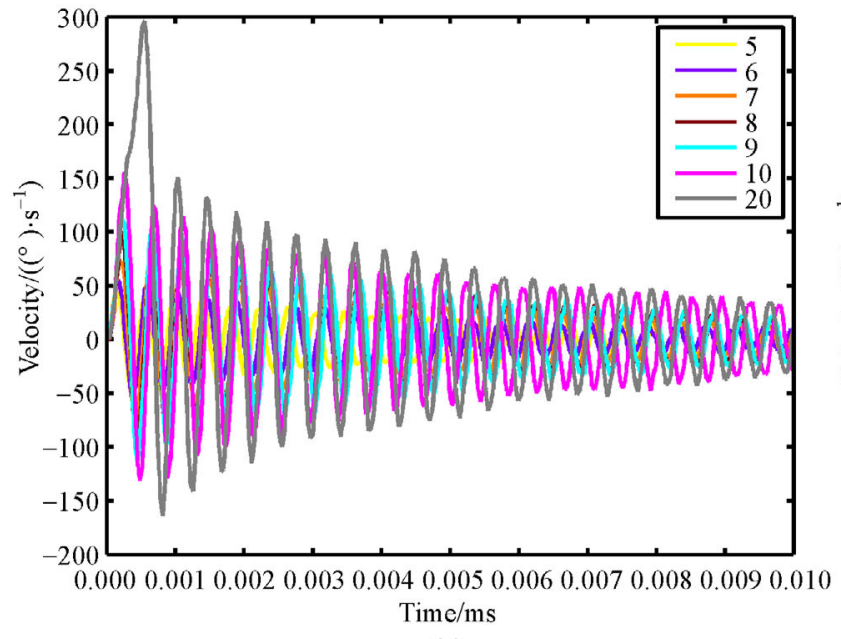

(a)

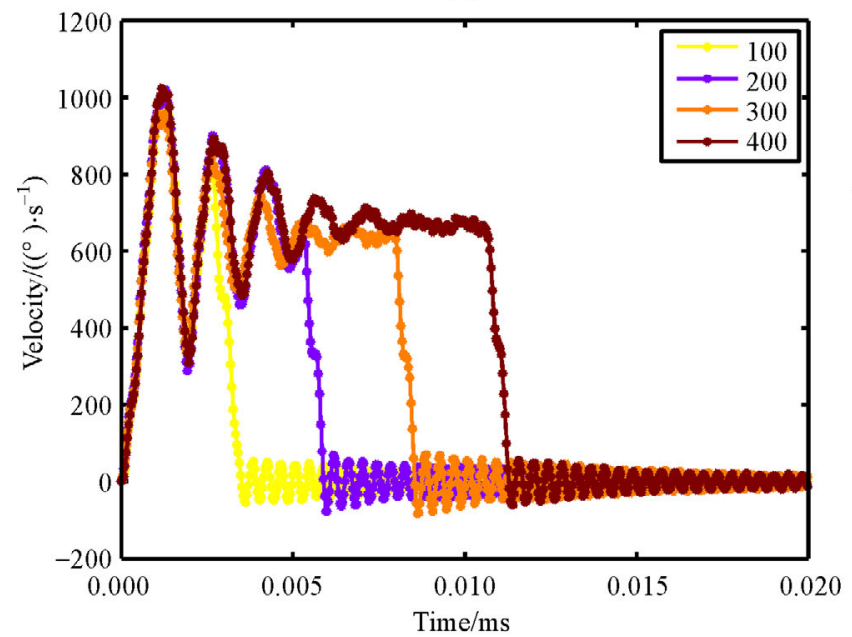

(c)

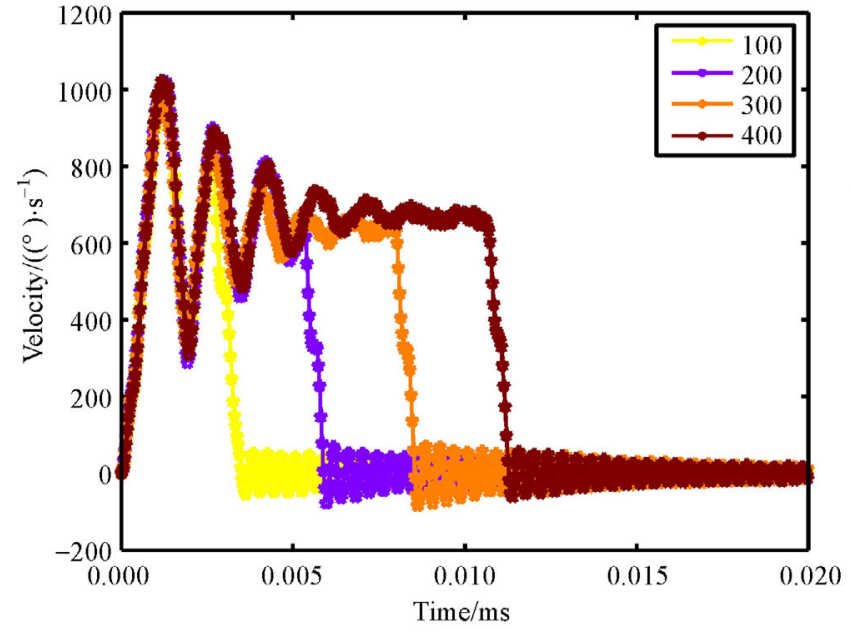

(e)

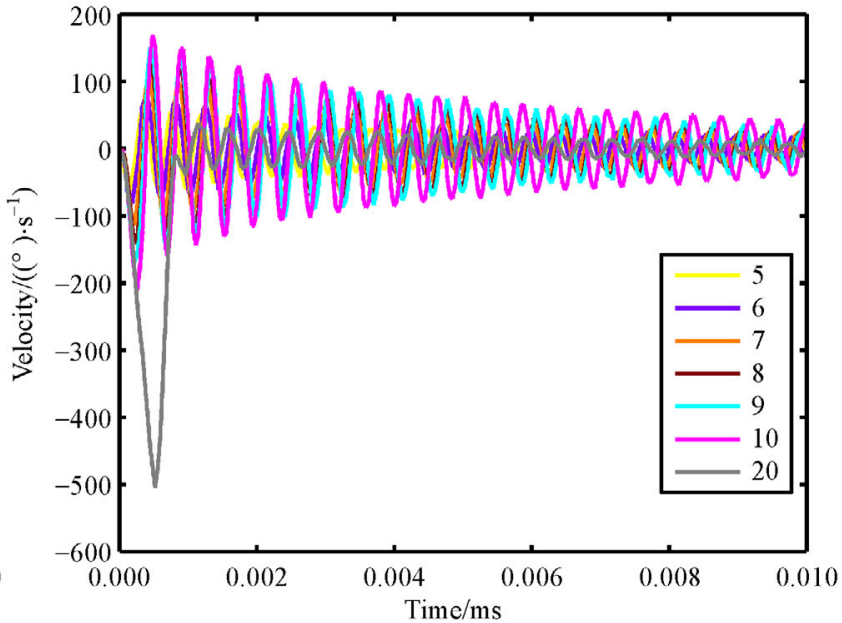

(b)

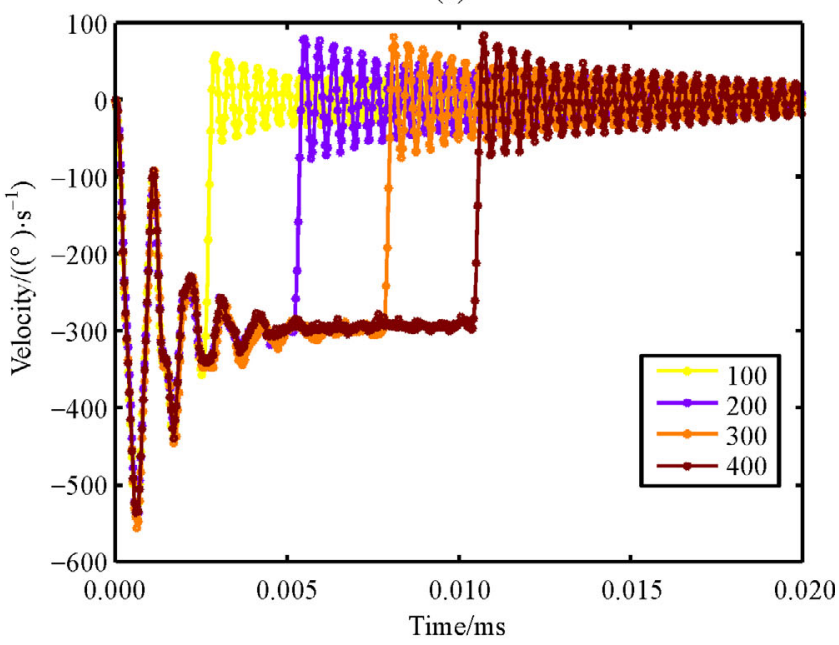

(d)

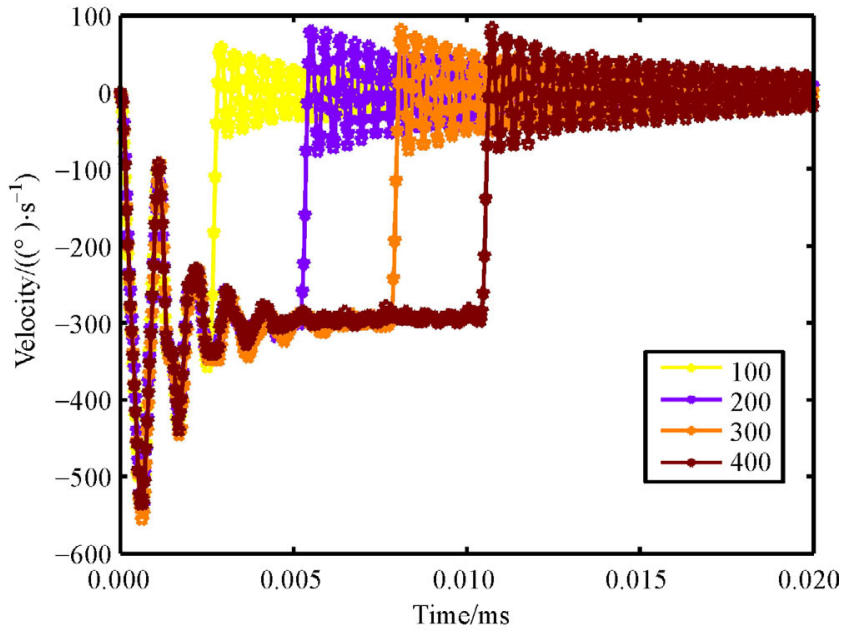

(f)

Fig. 10 Velocity with different pulse numbers: (a) Pulse number is from 5 to 20 in CW direction; (b) pulse number is from 5 to 20 in CCW direction; (c) pulse number is from 30 to 90 in CW direction; (d) pulse number is from 30 to 90 in CCW direction; (e) pulse number is from 100 to 400 in $\mathrm{CW}$ direction; (f) pulse number is from 100 to 400 in $\mathrm{CCW}$ direction. 


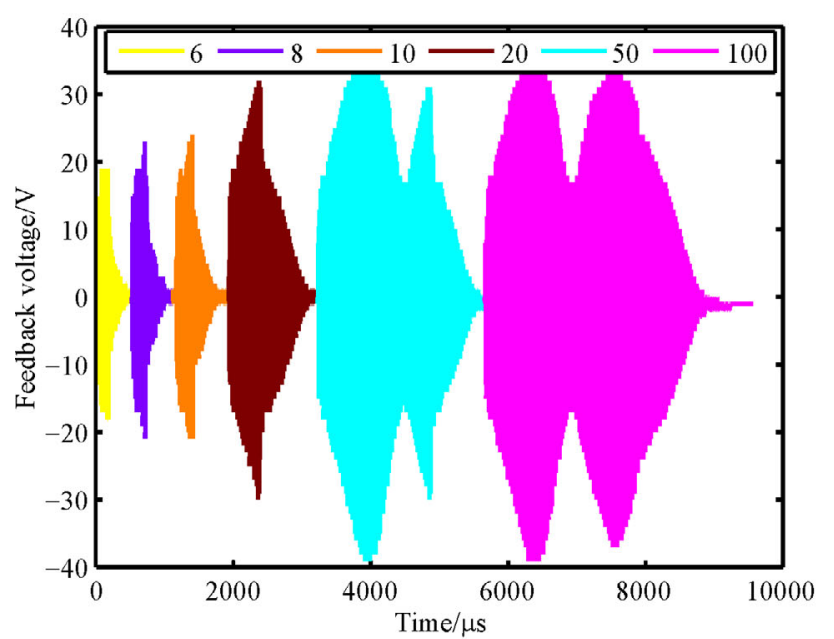

(a)

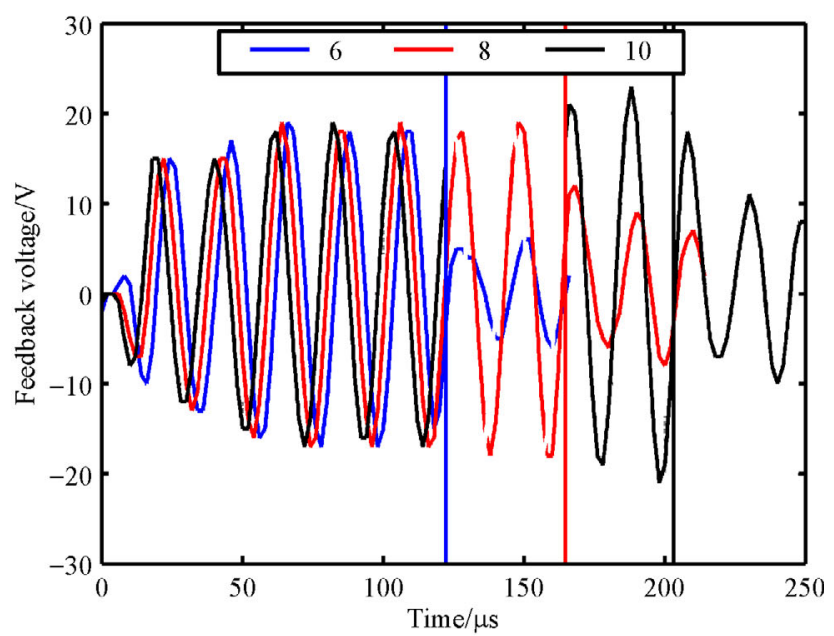

(b)

Fig. 11 Feedback voltages in function of different excitation numbers when the motor operates in CW direction. (a) Pulse number is from 6 to 100; (b) pulse number is from 6 to 10 .

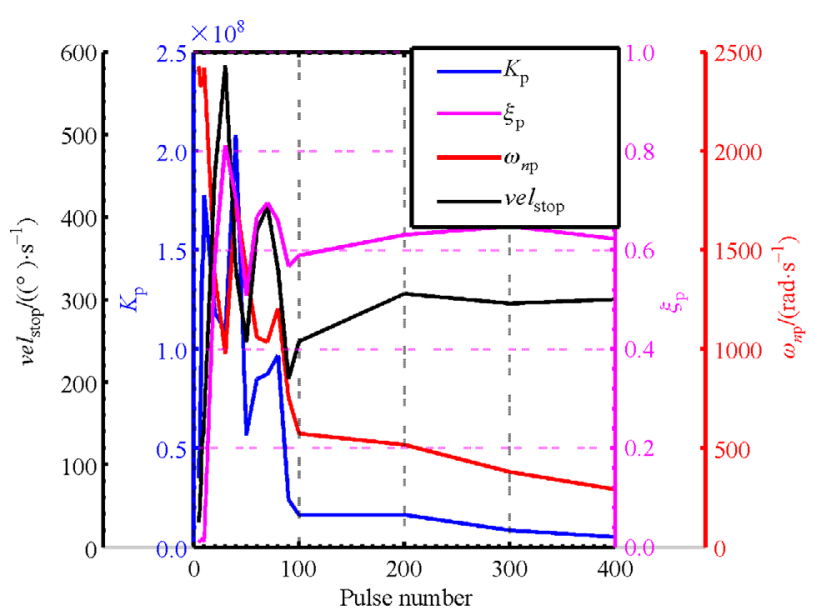

(a)

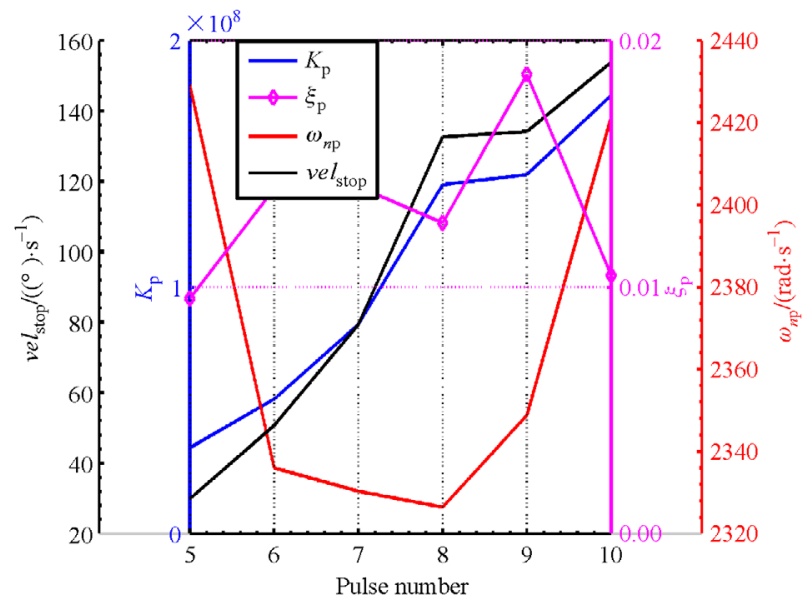

(c)

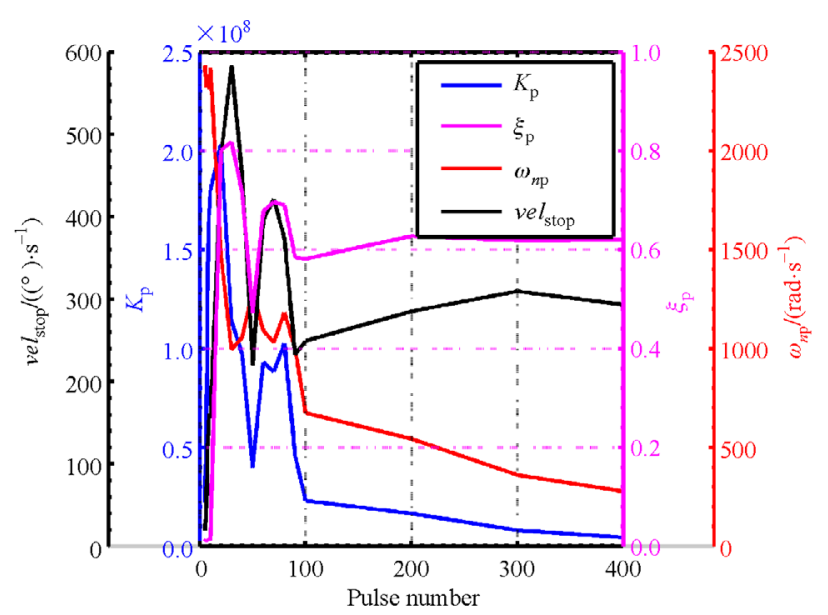

(b)

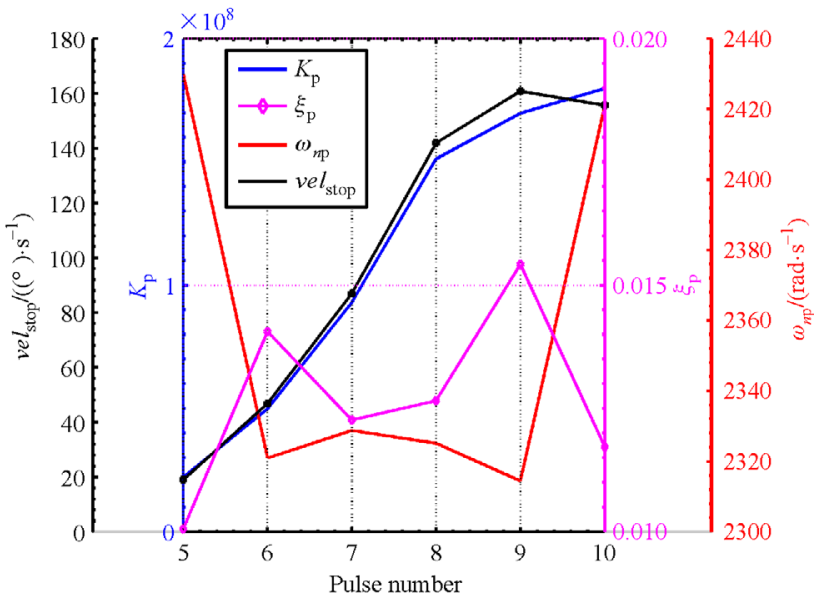

(d)

Fig. 12 Identified parameters of stopping process: (a) Pulse number is from 5 to 400 in CW direction; (b) pulse number is from 5 to 400 in $\mathrm{CCW}$ direction; (c) pulse number is between 5 and 10 in $\mathrm{CW}$ direction; (d) pulse number is between 5 and 10 in $\mathrm{CCW}$ direction. 
Table 1 Bidirectional identified parameters of startup process

\begin{tabular}{lcccc}
\hline Direction & Amplitude/V & Frequency/kHz & $\xi_{\mathrm{s}}$ & $\omega_{n s} /\left(\mathrm{rad} \cdot \mathrm{s}^{-1}\right)$ \\
\hline $\mathrm{CW}$ & 200 & 43 & 0.1099 & 947.307 \\
$\mathrm{CCW}$ & 200 & 43 & 0.1115 & 933.827 \\
\hline
\end{tabular}

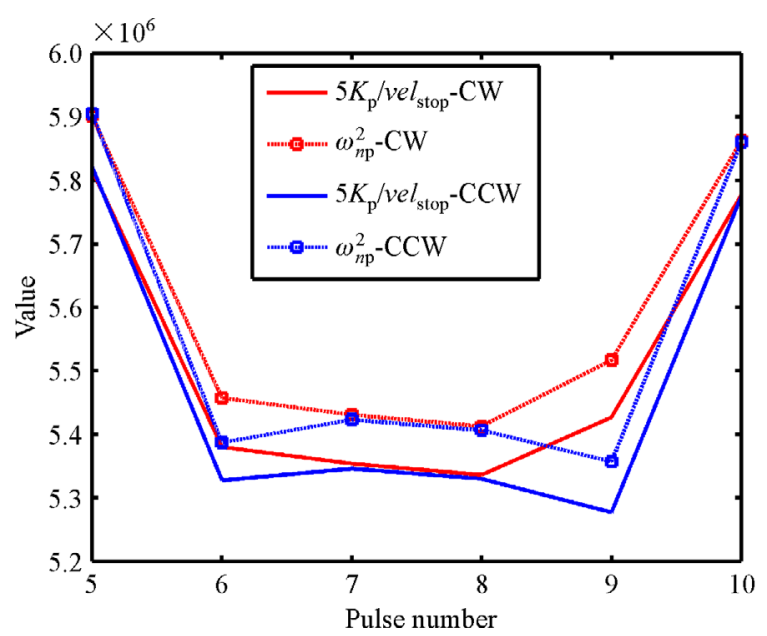

Fig. 13 Values of $5 K_{\mathrm{p}} / v e l_{\text {stop }}$ and $\omega_{n \mathrm{p}}^{2}$ in both directions.

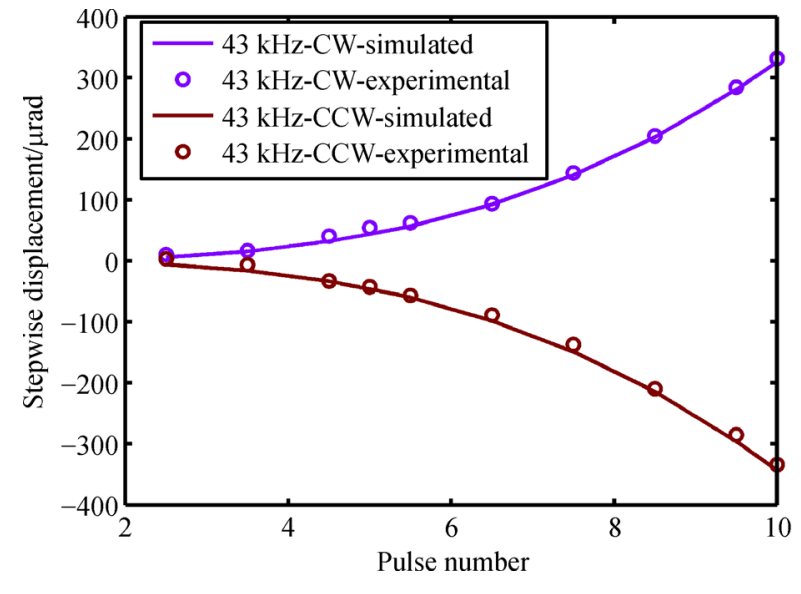

Fig. 14 Fitting results with different pulse numbers.

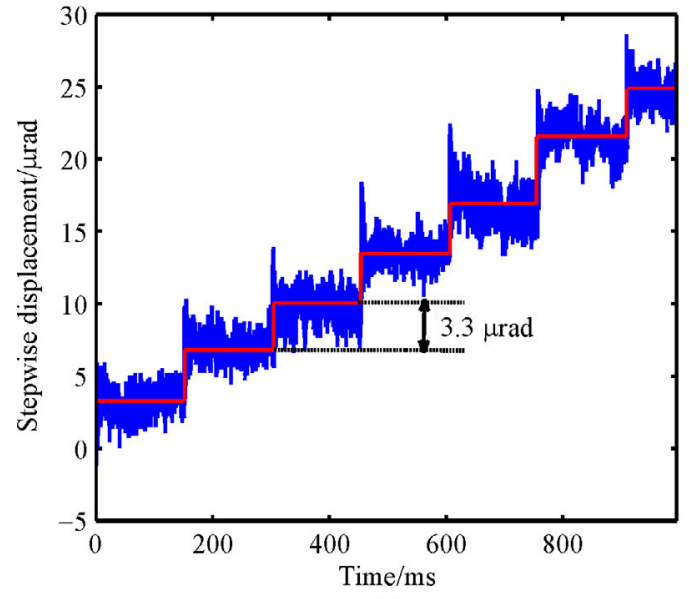

(a)

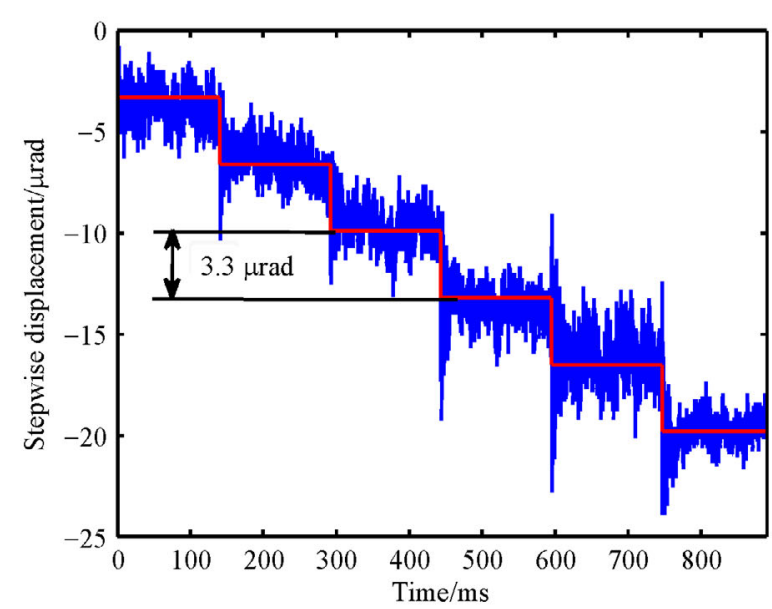

(b)

Fig. 15 Stepwise displacements (200 V, $43 \mathrm{kHz}$ ): (a) $\mathrm{CW}$ direction; (b) $\mathrm{CCW}$ direction.

practical applications. When amplitude is $200 \mathrm{~V}$ and frequency is $43 \mathrm{kHz}$, the available and reliable pulse number for the precise stepwise motion is no more than 2 for the case of PUM60.

\section{Conclusions}

The TRUM shares the merits of the precise angular displacement due to the piezoelectric actuation with controllable pulses. The modal function, mechatronic model, and synchronous experimental system are applied to evaluate the laws of the stepwise characteristics of openloop precise movement. The startup-shutdown stage is selected as the unit of the stepping movement. Different from the previous study, the present study investigates the kinematic and dynamic laws of the stopping stage. The derived equations demonstrate that at the shutdown stage, either the upper bound or the lower bound of the modal amplitude is connected to the modal displacement and speed at the shutdown moment. Therefore, an elaborate relationship exists between the attenuation in the functions of different pulse numbers. When the pulse number is small, the accumulating angle after the shutdown moment 
cannot be non-negligible. The identification based on the proposed two-stage two-order transfer functions is implemented, and we find the stable domain of the pulse number, where $2 \leqslant N \leqslant 10$ when the driving amplitude and frequency are $200 \mathrm{~V}$ and $43 \mathrm{kHz}$, respectively. The identified results when the pulse numbers are no more than 10 show good fitness with the experimental results, which verifies the validity of the simplified model. Finally, the precise stepping resolution $(3.3 \mu \mathrm{rad})$ of both directions are achieved by selecting the specific pulse numbers.

In addition, this study finds the differences in the vibration states and the stepwise displacement between $\mathrm{CW}$ and $\mathrm{CCW}$ directions, such as the speed unbalance phenomenon recorded in Ref. [35]. The displacement increments and vibration amplitude in the $\mathrm{CCW}$ direction are larger than those in the $\mathrm{CW}$ direction, which may originate from manufacturing and assembly error. However, this assumption is overgeneralized and cannot form guidelines for motor manufacturing or control. In the future, further in-depth mechanism analysis and compensation strategy will be conducted for precise position control in several applications.

Acknowledgements The authors acknowledge the financial support from the National Basic Research Program of China (973 Program) (Grant No. 2015CB057503). The authors declare no conflict of interest.

Open Access This article is licensed under Creative Commons Attribution 4.0 International License, which permits use, sharing, adaptation, distribution, and reproduction in any medium or format, as long as appropriate credit is given to the original author(s) and the source, a link is provided to the Creative Commons license, and any changes made are indicated.

Images or other third-party materials in this article are included in the article's Creative Commons license, unless indicated otherwise in a credit line to the material. If material is not included in the article's Creative Commons license and your intended use is not permitted by statutory regulation or exceeds the permitted use, you will need to obtain permission directly from the copyright holder.

To view a copy of this license, visit http://creativecommons.org/licenses/ by/4.0/.

\section{References}

1. Xu D, Liu Y, Shi S, et al. Development of a non-resonant piezoelectric motor with nanometer resolution driving ability. IEEE/ ASME Transactions on Mechatronics, 2018, 23(1): 444-451

2. Xu D, Liu Y, Liu J, et al. Developments of a piezoelectric actuator with nano-positioning ability operated in bending modes. Ceramics International, 2017, 43: S21-S26

3. Huang W, Tao J, Sun M, et al. Modeling and experiment of precision rotary positioner with large stroke driven by non-resonant piezoelectric motor. Optics and Precision Engineering, 2016, 24(11): 2712-2720 (in Chinese)

4. Chen X, Huang W, Lu Q, et al. Working mechanism of a kind of non-resonant linear piezoelectric motor with flexible driving end. Transactions of Nanjing University of Aeronautics and Astronautics, 2018, 35(5): 749-759
5. Wang L, Liu Y, Li K, et al. Development of a resonant type piezoelectric stepping motor using longitudinal and bending hybrid bolt-clamped transducer. Sensors and Actuators A: Physical, 2019, 285: 182-189

6. Zhang Q, Chen W, Liu Y, et al. A frog-shaped linear piezoelectric actuator using first-order longitudinal vibration mode. IEEE Transactions on Industrial Electronics, 2017, 64(3): 2188-2195

7. Liu J, Liu Y, Zhao L, et al. Design and experiments of a single-foot linear piezoelectric actuator operated in stepping mode. IEEE Transactions on Industrial Electronics, 2018, 65(10): 8063-8071

8. Shi W, Zhao H, Ma J, et al. Dead-zone compensation of an ultrasonic motor using an adaptive dither. IEEE Transactions on Industrial Electronics, 2018, 65(5): 3730-3739

9. Jin J, Zhao C. Linear ultrasonic motor using quadrate plate transducer. Frontiers of Mechanical Engineering in China, 2009, 4(1): 88-91

10. Song L, Shi J Z. Nonlinear Hammerstein model of ultrasonic motor for position control using differential evolution algorithm. Ultrasonics, 2019, 94: 20-27

11. Abdullah M, Takeshi M. Efficiency optimization of rotary ultrasonic motors using extremum seeking control with current feedback. Sensors and Actuators A: Physical, 2018, 289: 26-33

12. Shi S, Chen W, Liu J, et al. Ultrasonic linear motor using the L-B mode Langevin transducer with an exponential horn. Frontiers of Mechanical Engineering in China, 2008, 3(2): 212-217

13. Zhang H, Shi Y, Zhao C. Precision control system of two-DOF stage with linear ultrasonic motor. Frontiers of Mechanical Engineering in China, 2008, 3(4): 421-425

14. Mohd Romlay F R, Wan Yusoff W A, Mat Piah K A. Increasing the efficiency of traveling wave ultrasonic motor by modifying the stator geometry. Ultrasonics, 2016, 64: 177-185

15. Jin J, Zhao C. Bi-modes alternation stepping ultrasonic motors. Frontiers of Mechanical Engineering in China, 2008, 3(1): 101-105

16. Wang G Q, Tan J P, Zhao Z X, et al. Mechanical and energetic characteristics of an energy harvesting type piezoelectric ultrasonic actuator. Mechanical Systems and Signal Processing, 2019, 128: 110-125

17. Liang W, Ma J, Tan K K. Contact force control on soft membrane for an ear surgical device. IEEE Transactions on Industrial Electronics, 2018, 65(12): 9593-9603

18. Flueckiger M, Bullo M, Chapuis D, et al. FMRI compatible haptic interface actuated with traveling wave ultrasonic motor. In: Proceedings of IEEE Industry Applications Conference Fortieth IAS Annual Meeting. Hong Kong: IEEE, 2005

19. Chapuis D, Gassert R, Burdet E, et al. Hybrid ultrasonic motor and electrorheological clutch system for MR-compatible haptic rendering. In: Proceedings of IEEE/RSJ International Conference on Intelligent Robots \& Systems. Beijing: IEEE, 2006

20. Kandare G, Wallaschek J. Derivation, and validation of a mathematical model for traveling wave ultrasonic motors. Smart Materials and Structures, 2002, 11(4): 565-574

21. Boumous Z, Belkhiat S, Kebbab F Z. Effect of shearing deformation on the transient response of a traveling wave ultrasonic motor. Sensors and Actuators A: Physical, 2009, 150(2): 243-250

22. Nakagawa Y, Saito A, Maeno T. Nonlinear dynamic analysis of traveling wave-type ultrasonic motors. IEEE Transactions on 
Ultrasonics, Ferroelectrics, and Frequency Control, 2008, 55(3): 717-725

23. Nakamura K, Kurosawa M, Kurebayashi H, et al. An estimation of load characteristics of an ultrasonic motor by measuring transient responses. IEEE Transactions on Ultrasonics, Ferroelectrics, and Frequency Control, 1991, 38(5): 481-485

24. Shen S, Huang W, Zhao C. Wavelet transform applied to test and analysis on starting-up and stopping responses of the ultrasonic motor. In: Proceedings of IEEE Symposium on Ultrasonics. Honululu: IEEE, 2003

25. Wu X, Hua L, Qiang Y, et al. Studies on stepping characteristics of the traveling-wave ultrasonic motor. In: Proceedings of 2011 International Conference on Electronics Optoelectronics. Dalian: IEEE, 2011

26. Zhao C. Ultrasonic Motors: Technologies and Applications. Beijing: Springer, 2011

27. El Ghouti N. Hybrid modeling of a traveling eave piezoelectric motor. Dissertation for the Doctoral Degree. Aalborg: Aalborg University, 2000, 95-177

28. Shi W, Zhao H, Ma J, et al. Optimal working frequency of ultrasonic motors. Ultrasonics, 2016, 70: 38-44

29. Shi W, Zhao H, Ma J, et al. An optimum-frequency tracking scheme for ultrasonic motor. IEEE Transactions on Industrial Electronics, 2017, 64(6): 4413-4422

30. Li S Y, Ou W C, Yang M, et al. Temperature evaluation of travelingwave ultrasonic motor considering the interaction between temperature rise and motor parameters. Ultrasonics, 2015, 57: 159-166

31. NI Inc. NI R Series Multifunction RIO Specification. Integrated Analog and Digital I/O with FPGA Technology. 2014

32. Vezzoli E, Vidrih Z, Giamundo V, et al. Friction reduction through ultrasonic vibration Part 1: Modelling intermittent contact. IEEE Transactions on Haptics, 2017, 10(2): 196-207

33. Sednaoui T, Vezzoli E, Dzidek B M, et al. Friction reduction through ultrasonic vibration Part 2: Experimental evaluation of intermittent contact and squeeze film levitation. IEEE Transactions on Haptics, 2017, 10(2): 208-216

34. Storck H, Littmann W, Wallaschek J, et al. The effect of friction reduction in the presence of ultrasonic vibrations and its relevance to traveling wave ultrasonic motors. Ultrasonics, 2002, 40(1-8): 379383

35. Chen Z H, Zhao C C, Huang W Q. An effective frequency tracking control and balancing compensation between $\mathrm{CW} \& \mathrm{CCW}$ rotation speed techniques for the ultrasonic motor. In: Proceedings of Ultrasonics Symposium. Monreal: IEEE, 2004 